\title{
L'ASCESA DELL'EUROPA MERIDIONALE: CENTRO E PERIFERIA NELLA COMUNITÀ EUROPEA
}

\author{
di Robert Leonardi
}

\section{Le contraddizioni tra realtà e teoria}

La ratifica del Trattato di Maastricht tra i dodici paesi membri della Comunità europea mette in evidenza una contraddizione sottesa agli studi e alle riflessioni sull'Europa meridionale. In passato gli stati di questa area - Grecia, Spagna, Portogallo e anche l'Italia - sono stati descritti come paesi periferici o semi-periferici. Nelle analisi delle loro strutture politiche ed economiche si è sottolineata la difficoltà che questi paesi hanno incontrato nell'inserirsi nel flusso dello sviluppo economico europeo, nel partecipare attivamente alla determinazione delle politiche all'interno dell'Europa e alla definizione della posizione europea sulle problematiche mondiali. La gestione delle vicende europee continua ad essere vista come un processo dominato dai paesi settentrionali, cioè da quei paesi che hanno una più antica tradizione di istituzioni democratiche e di economie robuste.

Se, tuttavia, esaminiamo più attentamente i recenti avvenimenti in Europa, queste generalizzazioni non sembrano più rispecchiare la realtà. Al vertice di Edimburgo il ruolo della Spagna e dei paesi periferici si è rivelato centrale per giungere a un accordo sulla proposta per il bilancio Delors II e a una soluzione di compromesso riguardo alla possibilità concessa alla Danimarca di non attuare le politiche europee per la cittadinanza e per la difesa. Inoltre, il processo di ratifica del Trattato di Maastricht ha dimostrato come siano proprio i paesi settentrionali ad opporre le maggiori resistenze - a livello sia governativo che popolare - all'avvio della transizione verso una piena unione economica e politica. Di converso, gli stati e le popolazioni dei paesi meridionali e periferici continuano ad esprimere un diffuso appoggio verso il processo di integrazione europea. Perché? 
Secondo le teorie sui rapporti tra centro e periferia, l'integrazione economica e politica non è nell'interesse dei paesi e delle regioni di periferia. La dicotomia tra centro e periferia, da una parte, e i costi e i benefici dell'integrazione, dall'altra, sono tematiche comuni negli studi di scienza politica e di economia sull'integrazione.

Nel corso degli anni ottanta l'attenzione per la trasformazione politica ed economica dell'Europa meridionale è cresciuta in misura notevole grazie alla convergenza di tre fenomeni indipendenti: 1) l'instaurazione di regimi democratici (dal 1974 al 1977 i regimi autoritari in Portogallo, Grecia e Spagna sono stati sostituiti da democrazie parlamentari); 2) l'ascesa al potere alla fine degli anni settanta e all'inizio degli anni ottanta di governi a guida socialista (Portogallo e Grecia, ma anche Italia e Francia); 3) lo sviluppo economico, con tassi di crescita superiori a quelli dei paesi settentrionali. Come hanno osservato Hudson e Lewis, nel corso degli ultimi due decenni i tassi di crescita nell'Europa meridionale «sono stati superiori a quelli della maggior parte dei paesi a capitalismo avanzato, specie nel settore manifatturiero, mentre l'Europa meridionale diventava più importante nella divisione internazionale del lavoro» (1985, 35). Analogamente, Allan Williams poneva il problema dello sviluppo economico nell'Europa del Sud in termini molto provocatori: «Prima degli anni cinquanta era corretto chiedere “perché è sottosviluppata l'Europa meridionale?". Ma ora questa domanda è stata sostituita da "come mai l'Europa meridionale si è sviluppata così rapidamente?"» (1974).

La crescita economica e lo sviluppo democratico nei sistemi politici del Sud Europa, cui si aggiunge l'adesione alla Comunità europea, hanno inficiato l'adeguatezza di svariate teorie tratte dal pensiero marxista classico e dall'esperienza di paesi del Terzo Mondo, quali le teorie della dipendenza e dell'estrazione del plusvalore ${ }^{1}$. Alla luce degli spettacolari tassi di crescita della Spagna a metà degli anni ottanta e del Portogallo alla fine dello stesso decennio, nonché del favore manifestato dalla cittadinan$\mathrm{za}$ in generale verso l'integrazione europea, questi approcci teorici ora sembrano superati.

Di fronte al contrasto tra il pessimismo che caratterizzava la

1 La manifestazione di questi due fenomeni non ha dissuaso gli studiosi dell'Europa meridionale dall'applicare le teorie della dipendenza nel tentativo di prevedere lo sviluppo dei paesi mediterranei (vedi Seers et al. 1979). 
pubblica opinione più informata nei confronti dell'Europa meridionale prima degli anni ottanta $\mathrm{a}^{2}$ e le trasformazioni politiche ed economiche che sono intervenute successivamente, come si fa a spiegare un cambiamento così netto con l'insieme di modelli teorici attualmente a disposizione? La tesi di questo saggio è che nel corso degli ultimi due decenni i paesi e le regioni dell'Europa meridionale sono stati testimoni di una trasformazione economica e sociale che ha unito, da una parte, l'apertura dei mercati nazionali e la loro integrazione con l'economia europea e, dall'altra, una maggiore democratizzazione e la fine dell'isolamento politico nell'ambito dell'Europa. Con le attuali teorie dell'economia e della scienza politica non è facile spiegare come questa combinazione di fattori abbia potuto dar luogo all'espansione economica, alla stabilizzazione e al consolidamento di processi democratici nella periferia dell'Europa meridionale e alla mobilitazione di un processo di sviluppo economico e politico.

Il tasso e il tipo di sviluppo economico nei paesi del Sud Europa negli anni ottanta sono tali da non poterlo più attribuire all'omogeneizzazione dei fattori economici creata dalla migrazione della forza lavoro e dalle rimesse dall'estero. Anzi, le migrazioni su larga scala per motivi di lavoro sono cessate, si sono registrati notevoli investimenti di capitali; i beni e servizi prodotti in questi paesi si sono affermati sui mercati europei. A differenza di ciò che accade oggi nell'Europa dell'Est, la trasformazione dell'Europa meridionale nel corso degli anni settanta e ottanta è un buon esempio di come i cambiamenti economici possano tenere il passo con i cambiamenti politici nel far progredire un paese verso un nuovo equilibrio politico ed economico. Nel caso dell'Europa meridionale questa transizione è stata facilitata e rafforzata dall'ingresso di Portogallo, Spagna e Grecia nella Comunità europea.

La seguente analisi di quello che è successo nell'Europa del Sud verrà presentata in due parti. Anzitutto si tratteranno i rapporti interni agli stati nazionali, così come sono concepiti dalle teorie su centro e periferia; poi si svolgerà una riflessione empirica sulle tendenze di sviluppo economico a livello regionale e nazionale.

${ }^{2}$ Sono di particolare interesse i lavori compiuti nell'ambito della scienza politica sulla stabilità dei sistemi multipartitici dell'Europa meridionale, i quali venivano visti come fonte di regimi politici instabili. Per una trattazione dei sistemi multipartitici e del pluralismo polarizzato, vedi Sartori (1976). 
La comparazione si incentrerà sulle prestazioni dei paesi meridionali da una parte e i primi nove paesi membri, cioè i sei fondatori della Cee e i tre stati che hanno aderito alla Comunità nel 1974, dall'altra.

A questa regola generale si oppongono due eccezioni. L'Irlanda, benché appartenga alla Comunità dal 1974 e non sia un paese meridionale, verrà trattata in sede di analisi come un paese periferico in quanto ha molte caratteristiche economiche in comune con i suoi vicini meridionali. Il Mezzogiorno d'Italia, inoltre, verrà preso come esempio di regione periferica nonostante l'Italia sia uno dei paesi fondatori della Comunità europea del carbone e dell'acciaio (Trattato di Parigi) e della successiva Comunità economica europea (Trattato di Roma). Le caratteristiche sociali ed economiche delle regioni meridionali italiane le rendono, per alcuni aspetti, più simili alla Spagna meridionale e al Portogallo che non all'Italia centrale e settentrionale.

\section{Centro e periferia in scienza politica e in economia}

Il lavoro di Rokkan e Urwin $(1982 ; 1983)$ e di Tarrow (1977) costituiscono un evidente punto di partenza nell'ambito della scienza politica per quanto attiene ai rapporti tra centro e periferia in Europa. Rokkan e Urwin coniugano un'attenzione particolare per lo stato nazionale a una trattazione complessiva delle trasformazioni in atto in tutta Europa nel corso dei secoli; Tarrow cerca di usare gli stessi concetti al fine di analizzare le relazioni tra centro e periferia in Francia e in Italia. In entrambi i casi l'obiettivo principale è formulare un modello formale di politica territoriale che possa tornare utile per l'analisi dell'evoluzione della struttura socio-economica dello spazio fisico in Europa - cioè della «distanza» tra centro e periferia - e dei riflessi politici che ne conseguono.

Le definizioni di «centro» e «periferia» adottate da questi autori mettono in rilievo alcuni problemi tipici delle analisi territoriali in Europa. Spesso la definizione dei due concetti rende impossibile il controllo empirico; queste definizioni vengono presentate piuttosto in termini di «tipi ideali», già gravidi delle conclusioni alle quali l'analisi dovrebbe giungere.

In entrambe le versioni del concetto il punto di partenza è la definizione del centro e l'individuazione di una serie di carat- 
teristiche strutturali. Nella definizione del centro ci si pone i seguenti quesiti (Rokkan e Urwin 1983, 6; Tarrow 1977, 18): dove si incontrano per negoziare e avviare processi decisionali coloro che detengono le risorse chiave?; dove si riuniscono per celebrare riti che affermano l'integrità del territorio e la particolare identità della popolazione?; dove hanno eretto i monumenti che simboleggiano questa identità?

$\mathrm{Nel}$ rispondere a queste domande gli autori sottolineano che l'ubicazione delle più importanti istituzioni militari, amministrative, economiche e culturali (cioè dei «centri di comando») offre l'indizio di maggiore rilievo circa l'individuazione dei centri territoriali. Dalla loro successiva analisi dei sistemi di stati nazionali in Europa, comunque, emerge che vi è una certa variazione da paese a paese nel grado di concentrazione dei centri decisionali di tipo politico, amministrativo, militare, economico e culturale.

Rokkan e Urwin individuano tre tipi di strutture territoriali, a seconda del grado di concentrazione dei maggiori organi decisionali. Il primo è la struttura monocefala, in cui vi è un'elevata concentrazione di istituzioni di comando in un'area ristretta. Nei centri monocefali i detentori delle risorse interagiscono tra di loro su brevi distanze, il che permette alle élites nazionali di condividere strutture per la comunicazione, l'intermediazione e l'esercizio del potere.

Il secondo tipo di struttura territoriale si distingue per la dispersione geografica dei diversi tipi di arena all'interno di una area molto ampia. Questo tipo di sistema si chiama policefalo e si caratterizza per «una segmentazione spaziale di diversi tipi di detentori di risorse e una catena di centri distinti, ognuno con un proprio profilo di gruppi elitari» (Rokkan e Urwin 1983, 6).

Il terzo tipo di struttura territoriale è misto: sul territorio del centro si concentrano diverse aree funzionali, ma il centro non ha il monopolio di tutte le funzioni. In passato, i processi di costruzione dello stato in questi paesi non sono riusciti ad estrarre tutte le funzioni dalla periferia e a centralizzarle in un unico centro geografico ${ }^{3}$.

Siccome ci sono diversi tipi di centro, è probabile che si

${ }^{3}$ In base ai criteri di Rokkan e Urwin, si possono raggruppare i paesi nelle categorie seguenti: paesi monocefali: Francia, Regno Unito, Belgio, Austria, Danimarca, Grecia, Irlanda, Portogallo; paesi intermedi: Svezia, Norvegia, Finlandia; paesi policefali: Germania, Italia, Paesi Bassi, Svizzera, Spagna. 
possano identificare anche diversi tipi di periferia. Infatti, Rokkan e Urwin identificano due tipi fondamentali di periferia: di interfaccia ed esterno. Le periferie di interfaccia si trovano in aree centrali e sono in grado di interagire con altri territori periferici (ad es., l'area lungo il confine orientale del Portogallo e il confine occidentale della Spagna; la Catalogna che interagisce con Languedoc-Roussillon; la regione Alpes-Côte d'Azur e la Liguria nel Mediterraneo settentrionale $)^{4}$. Con l'espressione «periferia esterna», invece, si denota un'area periferica priva di altri interlocutori territoriali al di là dei confini politici dello stato d'appartenenza a causa del carattere insulare, peninsulare o comunque esterno del proprio territorio (ad es., le regioni periferiche di Grecia, Irlanda, Italia e Regno Unito).

In entrambi $i$ casi, le regioni periferiche si trovano in una condizione di svantaggio rispetto al centro. Secondo Tarrow $(1977,15-38)$ le caratteristiche della periferia sono: 1$)$ la distanza fisica dal centro; 2) un accesso penalizzante alle risorse economiche, politiche e culturali; 3 ) la dipendenza dal centro per il proprio sostentamento e benessere. Nella maggior parte dei casi, le caratteristiche della periferia sono il risultato non di eventi casuali bensì di una precisa politica di sottomissione intrapresa dal centro. Rokkan e Urwin descrivono la periferia come un territorio penetrato, assimilato e dominato dal centro:

È spesso un territorio conquistato, come se fosse una sorta di colonia, amministrato da funzionari che rispondono più alle istruzioni di un centro geograficamente remoto che non ai desideri della periferia. Tenderà ad avere un'economia poco sviluppata - o un'economia di sussistenza esclusa dalla rete territoriale di scambi economici e di commercio, oppure un'economia monoprodotto la cui produzione viene venduta su piazze lontane - che quindi è molto vulnerabile alle frequenti fluttuazioni della domanda e dei prezzi, sui quali la periferia ha poco o nessun controllo. Infine, la periferia tenderà ad avere anche una cultura marginale: priva di istituzioni unificate e distinte proprie, la sua cultura sarà frammentaria e parrocchiale $(1983,2)$.

In modo analogo, le precedenti trattazioni della periferia vanno al di là della sola definizione geografica di perifericità. Come ha riconosciuto Tarrow, molte delle definizioni più diffuse di perifericità propongono un tipo ideale che potrebbe non

${ }^{4}$ Questi sono le aree di confine alle quali fa riferimento l'art. 10 del regolamento del Fondo europeo per lo sviluppo regionale del 1988, che promuove la cooperazione transfrontaliera. 
essere valido sempre e ovunque. La perifericità, in quanto caratteristica strutturale di un'area specifica, può esistere in tre diversi ambiti di vita sociale: politica, economia, cultura ${ }^{5}$. $\mathrm{Ma}$, a prescindere dall'ambito, la caratteristica fondamentale della perifericità è che essa dipende da uno o più centri e che la sua situazione e il suo futuro non possono essere contemplati distintamente da quelli del centro.

Selwyn (1979) descrive le caratteristiche economiche della periferia in modo simile: la periferia non è in grado di controllare l'uso delle proprie risorse; manca di innovazione locale; ha legami interni deboli; è dominata da flussi informativi che hanno origine al centro; la forza lavoro migra dalla periferia verso il centro. Man mano che l'economia periferica viene assorbita da quella centrale, la sua capacità di opporsi a una dipendenza strutturale dal centro si affievolisce. La periferia finisce per assoggettarsi al dominio del centro.

I concetti formulati nelle teorizzazioni su centro e periferia sono stati usati non solo per analizzare l'effetto dell'integrazione dei mercati all'interno degli stati nazionali ma anche nel contesto della Comunità europea. Seers si chiede «cosa ci guadagna un piccolo paese periferico e relativamente non industrializzato ad aderire a un sistema composto da paesi centrali tecnicamente avanzati?» $E$ risponde che, anche se nel breve periodo la periferia riceve vantaggi sotto forma di aumenti dei livelli di reddito mediante una strategia di migrazione della forza lavoro e di richiamo di capitali esteri, nel lungo periodo essa si assoggetta all'egemonia economica, militare e culturale del centro (1979, xviii). Seers ed altri studiosi dei rapporti economici tra centro e periferia (vedi, ad es., Overturf 1986) prevedono che dall'adesione alla Comunità europea trarranno beneficio capitalisti e lavoratori collegati alle multinazionali attive nei paesi periferici; ma l'apertura obbligata dei mercati periferici «distruggerà molte piccole imprese» $\mathrm{e}$ i settori impreparati (come quello tessile e dell'abbigliamento) ad affrontare «la piena forza competitiva delle grandi imprese del centro, nonostante i dislivelli retributivi» (Seers et al. 1979, 27).

Queste aspettative desunte dall'analisi dei rapporti tra centro e periferia rimandano a un insieme di condizioni economi-

${ }^{5}$ La considerazione della perifericità come una mera espressione di mobilitazione etnolinguistica nei confronti del centro rappresenta solo alcuni aspetti politici delle relazioni tra centro e periferia (vedi Urwin 1985). 
che, riportate qui di seguito, che possono essere sottoposte a controllo empirico mediante un loro confronto con l'effettiva storia dell'integrazione europea:

1. I tassi nazionali di crescita economica hanno sofferto in seguito all'apertura delle relative economie al commercio internazionale prima e all'integrazione nella Comunità europea poi?

2. Le piccole imprese della periferia europea sono state eliminate dalla concorrenza delle grandi imprese?

3. Le industrie manifatturiere dei paesi periferici hanno subìto dei danni a causa dell'apertura e dell'integrazione dei mercati nazionali?

4. È aumentata la migrazione della forza lavoro man mano che procedeva l'integrazione economica?

5. Si sono deteriorati i rapporti tra centro e periferia a livello comunitario e all'interno dei singoli stati?

Allo stesso modo, si potrebbero analizzare anche le conseguenze politiche dell'adesione da parte degli stati periferici del Sud a una comunità che si sta integrando politicamente:

1. Gli stati periferici sono diventati meno importanti nei processi decisionali riguardanti la distribuzione delle risorse comunitarie?

2. La periferia ha perso la capacità di controllare le decisioni attinenti alle proprie condizioni economiche e al proprio benessere socio-economico?

3. Nella determinazione delle posizioni espresse a nome dell'intera Comunità, ai paesi dell'Europa meridionale è stato riservato uno status politico subordinato?

Rokkan e Urwin e altri teorici dei rapporti tra centro e periferia non rispondono a queste domande e attribuiscono, invece, le trasformazioni economiche degli stati e delle regioni europee ad eventi politico-militari, alla scoperta di nuove rotte commerciali e a cambiamenti tecnologico-economici. Arrighi (1985) sostiene che nel corso degli ultimi quarant'anni la posizione delle economie periferiche dell'Europa nel sistema economico mondiale si è trasformata in modo marcato. Vi sarebbero stati, sempre secondo Arrighi, spostamenti di posizione significativi di Italia e di Spagna (da semi-periferici a centrali) e di Portogallo e di Grecia (da periferici a semi-periferici).

Sulla base delle analisi di Rokkan e Urwin (1983) e di Arrighi (1985), si possono individuare tre principali tendenze nella storia europea. Fino al XVI secolo l'Europa fu dominata dall'asse Est-Ovest di attività economiche e commerciali, fondato 
su una serie di centri commerciali importanti localizzati lungo la costa mediterranea. Questo asse cominciò ad orientarsi verso Nord con l'avvento della presenza musulmana nel Mediterraneo orientale, l'emergere del sistema degli stati nazionali, la scoperta del Nuovo Mondo e l'apertura delle rotte commerciali atlantiche. Il declino dell'asse Est-Ovest si accompagnò a un minore controllo sulle capitali nazionali settentrionali $\mathrm{da}$ parte delle città mediterranee.

La seconda principale tendenza riguarda la crescente importanza, nei secoli successivi, della dimensione Nord-Sud nell'economia europea. La Riforma facilitò in modo particolare questa tendenza: i legami tra stato e religione si fondarono in identità nazionali e furono ulteriormente rafforzati dagli inizi della rivoluzione industriale, per la quale il carbone fu la fonte di energia primaria e $i$ minerali ferrosi il fondamento per la produzione di beni strumentali. La rivoluzione industriale e l'introduzione della ferrovia come uno dei principali mezzi di trasporto interno resero ancora più importante il centro dello stato nazionale quale struttura di comando centralizzante per la conduzione degli affari politici ed economici nazionali e per la tutela della comunità politica dagli avversari interni ed esterni.

La centralizzazione raggiunse il culmine nel periodo tra le due guerre in un contesto di crescenti tensioni politico-militari tra gli stati nazionali europei. In seguito a processi sia interni sia internazionali, i paesi della periferia europea (Italia, Portogallo, Spagna e Grecia) si isolarono progressivamente dal resto dell'Europa e dall'economia mondiale al fine di realizzare obiettivi, ispirati internamente, di autarchia politica ed economica. In questo periodo, le autorità nazionali diedero maggiore importanza a considerazioni di stato che non di mercato, a soluzioni autarchiche tese a proteggere le attività economiche nazionali dagli effetti della concorrenza e della penetrazione internazionali.

Le tendenze autarchiche e centralizzanti subirono una netta inversione di rotta nel sistema europeo del secondo dopoguerra, man mano che i paesi furono obbligati ad aprire i rispettivi sistemi economici e politici. Ciò faceva parte delle condizioni strutturali imposte dagli Stati Uniti per la riorganizzazione della vita politica ed economica dell'Europa occidentale; le politiche commerciali, monetarie e di sicurezza furono demandate ad organizzazioni internazionali. In questo contesto di trasformazione, si ebbero anche alcuni slittamenti nei rapporti tra il centro dell'Europa settentrionale e la periferia meridionale. Al contra- 
rio di quanto era accaduto nel periodo interbellico, si dava un'importanza sempre minore al ruolo dello stato nella gestione dell'economia. Nel suo nuovo ruolo, lo stato aveva il compito di affiancare il mercato, non di sostituirlo. I governi autoritari cominciarono a cedere il passo ad istituzioni e procedure di democrazia parlamentare.

L'apertura delle economie nazionali ai capitali esteri, alla migrazione della forza lavoro e al commercio internazionale ebbe, all'inizio, un effetto sulla struttura dei rapporti sia interni che esterni tra centro e periferia. Alcuni dati citati da Rokkan e Urwin e da altri dimostrano che nel dopoguerra le differenze tra centro e periferia cominciarono a diminuire (Cuadrado Roura e Suarez-Villa 1992; Leonardi 1993b) e che le diminuzioni più nette nelle differenze socio-economiche tra centro e periferia ebbero luogo nei paesi del Sud Europa (Williams 1984; Arrighi 1985).

Una prima conferma a favore della tesi che le differenze tra centro e periferia sono in declino proviene da uno studio delle 97 regioni dei sei paesi fondatori della Cee, condotto da Biehl et al. (1972). Confrontando il prodotto interno lordo pro capite del 1960 con quello del 1969, risultò che le disparità regionali si erano attenuate. La loro analisi mostrò anche che il baricentro dell'economia europea si era spostato dal triangolo formato dalle regioni Ruhr, Wallonie e Nord Pas de Calais a un punto nodale ubicato lungo il basso Reno. A partire da questo centro, gli scaglioni di reddito digradavano muovendo verso il resto della Comunità, anche se la regolarità di questa struttura concentrica veniva turbata dalla presenza delle capitali nazionali e dei grandi centri urbani. Molle et al. (1980) sono giunti a conclusioni analoghe per il periodo che va dal 1950 al 1970.

Rokkan e Urwin, tuttavia, non hanno tenuto conto di questi risultati nella loro concezione dei rapporti centro-periferia, né hanno prestato molta attenzione all'effetto dell'integrazione europea sugli stati e sulle regioni periferiche. Le relazioni tra centro e periferia continuarono ad essere viste da Rokkan e Urwin secondo l'ottica dello stato nazionale e per lo più come una strada a senso unico: non hanno mai seriamente considerato la possibilità che la periferia potesse ottenere dall'integrazione tanto quanto il centro, se non di più.

Dati i loro assunti di partenza e i loro metodi di argomentazione basati su tipi ideali, le teorie sui rapporti tra centro e periferia rappresentano un approccio che è significativamente diverso da quello adottato dalla teoria funzionalista dell'integra- 
zione (Haas 1958; Lindberg 1963). Quest'ultima sottolinea il ruolo fondamentale dello sviluppo economico nell'alimentazione del processo di costruzione delle istituzioni e nella trasformazione delle relazioni politiche esistenti, ruolo che pertanto finisce per modificare lo stato nazionale. Le teorie sui rapporti tra centro e periferia nella scienza politica sono l'equivalente delle teorie dello squilibrio e della divergenza nell'economia: entrambi questi gruppi di teorie giungono alla conclusione che le implicazioni dell'integrazione economica e politica sono più negative che positive per la periferia.

La realtà empirica, tuttavia, indica l'esatto contrario. I paesi periferici considerano il passaggio a mercati e soluzioni istituzionali integrati e di maggiori dimensioni come un processo che comporta più vantaggi che svantaggi. Al fine di rendere conto di questo risultato è necessario prendere in esame un modello alternativo, di tipo convergente, dell'integrazione, basato su tipi empirici, non ideali, di definizione del centro e della periferia e dei processi mediante i quali questi ultimi interagiscono.

\section{Il modello convergente di integrazione europea}

Per poter capire quali siano le conseguenze dell'integrazione europea per i paesi dell'Europa meridionale è necessario riformulare i quesiti iniziali posti da Rokkan e Urwin per definire il centro di uno stato nazionale: dove si trovano i luoghi importanti dell'autorità del sistema europeo? È evidente che non esiste un centro monocefalo nel caso della Comunità europea. Per volontà comune, la Comunità è attualmente un'entità policefala in cui i centri dei processi decisionali politici, delle attività economiche e delle manifestazioni culturali sono stati consapevolmente distribuiti su tutto il suo territorio. Inoltre, la Comunità possiede periferie sia di interfaccia sia di tipo esterno. È necessario, pertanto, tenere conto di questi aspetti del processo di costruzione della Comunità nell'operativizzazione dei concetti che verranno impiegati nei controlli empirici.

La Comunità europea può essere concepita come un insieme variegato di processi mediante i quali il centro e la periferia interagiscono 6 . Nella fig. 1 è riportato un modello che contiene

${ }^{6} \mathrm{Nel} 1958$ Haas definì l'integrazione come un processo piuttosto che come una condizione. 


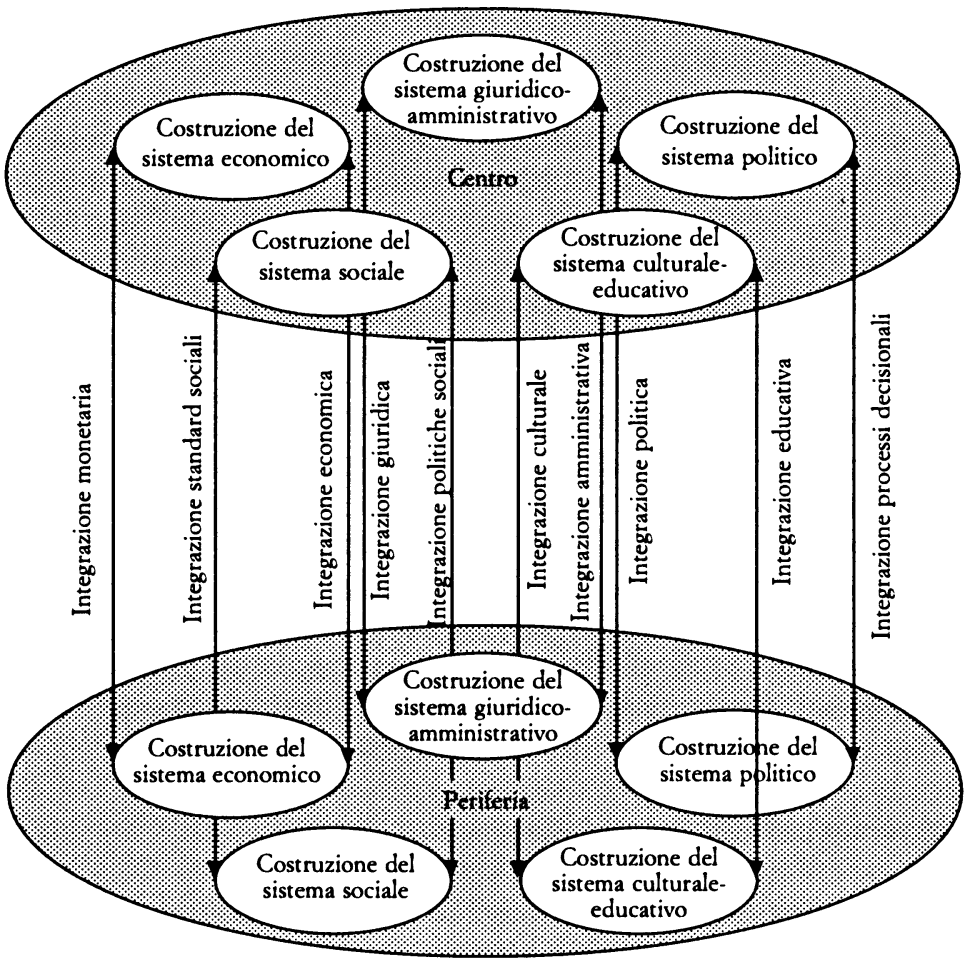

FIG. 1. Un modello di convergenza europea

le cinque componenti del processo di costruzione delle istituzioni così come si manifestano nella Comunità (cioè la creazione di nuove istituzioni politiche, economiche e giuridiche a livello europeo). Si possono studiare questi processi analizzando la trasformazione delle relazioni tra il «centro» comunitario e gli stati europei periferici, nonché delle strutture che regolano i legami tra centro e periferia all'interno dei singoli stati. A sua volta, ciascuna componente è caratterizzata da un processo di integrazione che può essere rilevato empiricamente nel corso del tempo al fine di accertare se la «distanza» o la «differenza» di dimensioni e di livello del rapporto centro-periferia stia aumentando o diminuendo.

La presenza di due frecce che collegano il «centro» e la «periferia» (fig. 1) indica la possibilità teorica che il processo di 
integrazione dia luogo a forze o ad esiti alternativi. La prima forza è la penetrazione della periferia da parte del centro così come viene concepita nelle teorie sui rapporti centro-periferia, mentre la seconda riguarda la possibilità che l'integrazione permetta alla periferia di accedere al centro, o di penetrarlo - in altre parole, offra, nel lungo periodo, vantaggi politici ed economici considerevoli alla periferia - e ponga fine allo status marginale e periferico degli stati nazionali esterni dell'Europa. Questa seconda alternativa - di penetrazione del centro da parte della periferia e di integrazione dal basso - non è contemplata né dalle teorie sui rapporti centro-periferia, né dalle teorie funzionaliste dell'integrazione. Haas e i suoi collaboratori affermano che l'integrazione è - più che un processo basato sulla mobilitazione di masse in favore di un'integrazione dal basso un processo elitario, incentrato su una relazione bilaterale in cui si incontrano la Comunità, da una parte, ed élites nazionali in ambito politico, amministrativo e economico dall'altra (Haas 1958). E tuttavia possibile, in termini sia teorici che pratici, che l'integrazione conferisca agli stati periferici e ai movimenti di base una possibilità di accesso e un'influenza nella determinazione degli esiti politici europei che non erano concepibili nel precedente contesto di negoziazione tra stati.

Ciascuno di questi ambiti di costruzione del sistema comunitario può essere operativizzato secondo le modalità evidenziate nella tab. 1 . In molti casi questi processi di convergenza vengono già racchiusi nei processi di costruzione dei programmi, delle attività e degli accordi intrapresi dalla Comunità al fine di promuovere l'integrazione a livello europeo in ambiti tematici specifici. L'aspettativa sottesa ai processi illustrati nella fig. 1 e nella tab. 1 è che le trasformazioni di tipo economico, politico, amministrativo e sociale che si avvereranno nel corso dell'integrazione europea andranno nella direzione di una maggiore coesione (cioè, saranno accompagnate da una riduzione delle differenze e degli squilibri) tra i paesi e le regioni della Comunità, così come prevedono la teoria della convergenza economica e la teoria neofunzionalista dell'integrazione.

Per motivi di spazio, ci limiteremo alla presentazione di indizi empirici attinenti alla periferia meridionale dell'Europa al fine di controllare i processi di integrazione riguardanti le tendenze nei dislivelli socio-economici nella Comunità. Cercheremo di dimostrare che la convergenza caratterizza anche altri ambiti di azione politica e amministrativa e si inserisce nel processo 
TAB. 1. Ambiti di differenza tra paesi e regioni centrali e periferici in Europa e risposte integrative della Comunità europea

\begin{tabular}{ll}
\hline Ambiti & Risposta comunitaria \\
\hline $\begin{array}{l}\text { Distanza fisica: tempo impiegato per } \\
\text { giungere dalla periferia al centro } \\
\text { per via aerea, ferroviaria, viaria } \\
\text { e con mezzi di telecomunicazione }\end{array}$ & $\begin{array}{l}\text { Reti di trasporti e } \\
\text { programmi di telecomunicazioni }\end{array}$ \\
$\begin{array}{l}\text { Differenze economiche: aumenti del Pil, } \\
\text { livelli di produttività, attività } \\
\text { manifatturiera, commercio, investimenti, etc. }\end{array}$ & Mercato unico \\
$\begin{array}{l}\text { Differenze monetarie: tassi di interesse, } \\
\text { tassi di cambio, entità del disavanzo } \\
\text { pubblico, propensione al risparmio }\end{array}$ & Unione monetaria europea \\
$\begin{array}{l}\text { Differenze politiche: programmi e campagne } \\
\text { elettorali dei partiti, norme elettorali, } \\
\text { contestazione dei risultati elettorali da } \\
\text { parte dei partiti, esiti elettorali, etc. }\end{array}$ & $\begin{array}{l}\text { Convergenza e coordinamento } \\
\text { dei programmi politici per le } \\
\text { elezioni del Parlamento europeo }\end{array}$
\end{tabular}

Procedure decisionali: governi di maggioranza o di coalizione, ruolo del consenso e del compromesso, ruolo dell'opposizione, etc.

Atto unico europeo e Trattato di Maastricht

Differenze culturali: preferenze artistiche e musicali, atteggiamenti sociali, valori

Differenze amministrative: strutture e procedure amministrative, reclutamento e avanzamento delle élites, legami con strutture subnazionali, coordinamento interministeriale, etc.

Differenze di politica sociale: livelli di spesa del settore pubblico per la disoccupazione, gli anziani, i giovani, le scuole materne, etc.

Differenze di condizione sociale: livelli di benessere e di consumo, speranza di vita, cause di morte, tassi di criminalità, etc.

Differenze di istruzione: livelli di analfabetismo, ordinamento dell'istruzione superiore, programmi di insegnamento nella scuola dell'obbligo, istituti di ricerca di punta

Differenze giuridiche: strutture e procedure giuridiche, procedura di appello, ruolo della Corte europea di giustizia e delle leggi comunitarie

Programma per le capitali culturali europee

Esperienze di cooperazione nel Comitato dei rappresentanti permanenti degli stati (Coreper) e comitati di esperti

Fondo sociale e Carta sociale

Carta sociale

Erasmus

Corte europea di giustizia e legislazione comunitaria 
che crea una definizione europea degli standard e dei processi che riguardano le élites politico-amministrative. Quando Haas (1975) cercò di valutare il grado di convergenza in Europa a metà degli anni settanta, la sua risposta fu ambigua e portò all'abbandono della teoria dell'integrazione. Oggi, in seguito all'Atto unico europeo, al Trattato di Maastricht e alla realizzazione del mercato unico, non è più accettabile una tale risposta.

$\grave{E}$ importante stabilire cosa sia davvero accaduto ai livelli di benessere socio-economico nei paesi periferici al fine di accertare se le previsioni avanzate dai teorici dei rapporti centro-periferia siano state confermate o smentite dagli esiti effettivi dell'integrazione europea. Sulla base del nostro modello multisettoriale della convergenza, ipotizziamo che i livelli socio-economici della periferia meridionale convergeranno con quelli dei membri settentrionali della Comunità.

Lo sviluppo economico delle regioni greche, spagnole e portoghesi (1981-1990)

Uno dei motivi più importanti per cui le popolazioni di Grecia, Spagna e Portogallo sono favorevoli all'adesione alla Comunità europea si basa sull'aspettativa che l'accesso alla Comunità offrirà uno stimolo positivo allo sviluppo delle economie nazionali e regionali mediante la creazione di mercati più ampi per beni prodotti localmente e azioni di richiamo per gli investimenti esteri nei loro settori manifatturieri e terziari. La tab. 2, mette in evidenza che $\mathrm{i}$ tassi di sviluppo relativi alle 34 regioni dei paesi che hanno aderito nel 1981 e nel 1986 reggono bene il confronto con quelli delle altre regioni della Comunità. I tassi di crescita per la Grecia dal 1975 al 1985 sono particolarmente degni di nota e non rispecchiano certo il quadro desolante che viene spesso evocato per descrivere la situazione di questo paese.

L'unica eccezione di rilievo a questa generalizzazione riguarda la regione di Attiki (cioè la regione ateniese), alla quale fanno capo oltre la metà della popolazione e la maggior parte del prodotto interno greco. Nel periodo preso in esame, infatti, l'Attiki ha avuto uno sviluppo molto modesto e persino di segno negativo. La sua prestazione deludente potrebbe essere dovuta a un'eccessiva saturazione delle attività economiche, accompagnata da una concentrazione dell'urbanizzazione e del 
TAB. 2. Variazioni percentuali annue medie del Pil pro capite (in Ecu, valori del 1985)

\begin{tabular}{|c|c|c|c|c|c|}
\hline Regione & $1975-85$ & 1985-89 & Regione & $1975-85$ & $1985-89$ \\
\hline Schleswig-Holstein & 1,6 & 3,5 & Zeeland & $-0,1$ & 4,1 \\
\hline Hamburg & 2,8 & 2,5 & Noordbrabant & 0,9 & 3,7 \\
\hline Niedersachsen & 2,4 & 3,2 & Limburg & 1,2 & 3,7 \\
\hline Bremen & 2,5 & 3,6 & & & \\
\hline Nordrhein-Westfalen & 1,8 & 2,8 & Vlaanderen & 2,1 & 3,0 \\
\hline Hessen & 2,3 & 3,2 & Bruxelles & 1,7 & 3,4 \\
\hline Rheinland-Pfalz & 2,1 & 3,0 & Wallonie & 1,7 & 3,9 \\
\hline Baden-Württemberg & 2,3 & 3,1 & & & \\
\hline Bavaria & 3,1 & 3,4 & Luxembourg & 2,2 & 3,3 \\
\hline Saarland & 3,0 & 2,3 & & & \\
\hline \multirow{2}{*}{ West Berlin } & 2,7 & 0,6 & North & 1,8 & 3,0 \\
\hline & & & York and Humberside & 1,8 & 2,6 \\
\hline Ile de France & 2,2 & 3,1 & East Midlands & 1,6 & 2,4 \\
\hline Champagne-Ardenne & 1,0 & 2,2 & East Anglia & 2,3 & 3,0 \\
\hline Picardie & 1,1 & 2,7 & Southeast & 2,2 & 4,1 \\
\hline Haute-Normandie & 1,8 & 1,8 & Southwest & 2,3 & 3,5 \\
\hline Centre & 2,3 & 2,4 & West Midlands & 1,5 & 3,2 \\
\hline Basse-Normandie & 2,3 & 0,7 & Northwest & 1,6 & 3,1 \\
\hline Borgogne & 2,0 & 2,8 & Wales & 1,5 & 3,4 \\
\hline Nord Pas de Calais & 1,0 & 2,2 & Scotland & 1,8 & 2,8 \\
\hline $\begin{array}{l}\text { Lorraine } \\
\end{array}$ & 0,7 & 2,7 & Northern Ireland & 1,5 & 2,7 \\
\hline Alsace & 1,9 & 3,2 & & & \\
\hline France-Comté & 1,1 & 2,1 & Ireland & 1,0 & 2,6 \\
\hline Pays de la Loire & 1,9 & 2,7 & & & \\
\hline Bretagne & 1,9 & 3,2 & Denmark & 2,4 & 0,8 \\
\hline Poitou-Carentes & 1,7 & 2,9 & & & \\
\hline Aquitaine & 2,5 & 1,4 & Anatoliki Makedonia & & \\
\hline Midi-Pyrénées & 2,6 & 2,0 & Thraki & 5,2 & 2,6 \\
\hline Limousin & 2,2 & 3,8 & Kentriki Makedonia & 2,1 & 2,6 \\
\hline Rhône-Alpes & 1,7 & 3,0 & Kytiki Makedonia & 1,1 & 2,6 \\
\hline Auvergne & 1,8 & 3,6 & Thessalia & 3,1 & 2,6 \\
\hline Languedoc-Roussillon & 1,9 & 2,9 & Ipeiros & 3,0 & 2,6 \\
\hline \multirow{3}{*}{$\begin{array}{l}\text { Provence-Alpes-Cote d'Azur } \\
\text { Corse }\end{array}$} & 3 & 16 & Ionia Nisia & 3,6 & 2,6 \\
\hline & 1,3 & 2,4 & Dykiti Ellada & 2,6 & 2,5 \\
\hline & & & Sterea Ellada & 2,4 & 2,6 \\
\hline Piemonte & 2,7 & 2,9 & Peloponnisos & 3,1 & 2,6 \\
\hline Val d'Aosta & 5,5 & 2,9 & Attiki & 0,4 & $-0,1$ \\
\hline Liguria & 7,5 & 3,5 & Voreio Aigaio & 3,1 & 2,5 \\
\hline Lombardia & 2,8 & & Notio Aigiao & 3,4 & 2,5 \\
\hline Trentino-Alto Adige & 3,0 & 4,2 & Kriti & 3,1 & 2,7 \\
\hline Veneto & 4,8 & 4,3 & & & \\
\hline Friuli-Venezia Giulia & 5,8 & 5,1 & $\begin{array}{l}\text { Gallcta } \\
\text { Asturias }\end{array}$ & 3,1 & $\begin{array}{l}4,2 \\
2,9\end{array}$ \\
\hline Emilia-Romagna & 0,3 & 3,8 & Cantabria & 1,8 & 0,9 \\
\hline Toscana & 2,9 & 2,4 & Pais Vasco & 0,5 & 3,9 \\
\hline Umbria & $-0,5$ & 2,0 & Navarra & $-0,1$ & 4,1 \\
\hline Lazio & 4,7 & 4,9 & La Rioja & 3,6 & 1,7 \\
\hline Campania & 3,0 & 1,2 & Aragon & 1,5 & 4,2 \\
\hline Abruzzo & 6,3 & 3,2 & Madrid & 0,6 & 4,1 \\
\hline Molise & 9,4 & 3,9 & Castilla y Leon & 2,0 & 3,3 \\
\hline Puglia & 3,9 & 4,1 & Castilla-La Mancha & $-0,9$ & 5,9 \\
\hline Basilicata & 4,1 & 5,8 & Extremadura & 7,4 & 2,3 \\
\hline Calabria & 1,2 & $-1,4$ & Cataluña & $-0,6$ & 4,6 \\
\hline Sicilia & 0,5 & 4,7 & Comunidad Valenciana & a $-0,1$ & 53 \\
\hline Sardegna & 3,1 & 4,0 & Baleares & 6,1 & 5,1 \\
\hline \multirow{7}{*}{$\begin{array}{l}\text { Groningen } \\
\text { Friesland } \\
\text { Drenthe } \\
\text { East Netherlands } \\
\text { Utrecht } \\
\text { Noordholland } \\
\text { Zuidholland }\end{array}$} & & & Andalucia & 0,5 & 4,1 \\
\hline & 0,8 & $\begin{array}{r}-6,3 \\
4,6\end{array}$ & Murcia & $-2,3$ & 5,8 \\
\hline & 3,1 & 0,7 & Norte & 1,6 & 3,9 \\
\hline & 0,8 & 2,9 & Centro & 4,5 & 5,4 \\
\hline & 0,7 & 2,1 & Lisboa e Vale do Tejo & 2,2 & 3,9 \\
\hline & 2,1 & 2,0 & Alentejo & 0,2 & 5,3 \\
\hline & 0,5 & 3,5 & Algarve & 0,1 & 4,5 \\
\hline
\end{tabular}


controllo politico in una sola area del territorio nazionale, quella intorno alla capitale. La Grecia è fortemente monocefala, e la città di Atene è per molti aspetti un buon esempio di un centro nazionale che ha impiegato le risorse in modo sempre più improduttivo. Ad Atene si è giunti al punto dei guadagni decrescenti. La prestazione scadente di Atene contribuisce in modo determinante al basso livello di reddito dell'intero paese, anche se la periferia esprime livelli soddisfacenti.

Un'altra spiegazione dei bassi tassi di sviluppo economico in Grecia rispetto agli altri nuovi paesi membri riguarda l'esiguità degli investimenti stranieri in Grecia. Prima di investire in Grecia, un'impresa estera deve accertarsi non solo che gli investimenti saranno redditizi ma anche che l'economia locale e il settore pubblico saranno capaci di garantire una serie di fattori essenziali.

Si potrebbe argomentare che in Grecia mancano sia fattori economici strutturali di qualità sia una burocrazia pubblica efficiente, capace di garantire procedure amministrative trasparenti e di soddisfare le esigenze espresse dagli operatori economici. La scarsa propensione degli investitori stranieri ad operare in Grecia e il basso livello di investimento interno potrebbero derivare più dalle incertezze attinenti alla trasparenza del processo politico e amministrativo che non dalla redditività degli investimenti stessi.

I dati riportati nella tab. 3 riguardano l'ammontare degli investimenti esteri diretti, le erogazioni dei fondi strutturali e la formazione di capitale nazionale fisso in quattro paesi periferici (Grecia, Spagna, Portogallo e Irlanda). Gli investimenti esteri in Grecia sono molto inferiori a quelli degli altri tre paesi: 3,15\% in Grecia contro un tasso più che doppio negli altri paesi. Se si confrontano le somme erogate dai fondi strutturali (fondi comunitari resi disponibili mediante i fondi regionali, sociali e agricoli) con il tasso di investimenti esteri diretti, risulta che il rapporto tra investimenti stranieri e fondi strutturali è molto diverso in Grecia rispetto agli altri tre paesi: in Grecia i fondi strutturali sono molto maggiori degli investimenti stranieri; negli altri tre paesi è il contrario. In Spagna gli investimenti stranieri erano già sette volte maggiori dei contributi comunitari durante il primo anno di adesione nel 1986 e sono rimasti elevati da allora. In Portogallo e in Irlanda si è avuto un maggiore equilibrio tra i due tipi di investimenti, ma la tab. 3 mostra che l'avvio dei programmi di investimento previsti dal quadro co- 
TAB. 3. Investimenti esteri diretti, erogazioni dei fondi strutturali comunitari e formazione di capitale fisso interno lordo nei paesi periferici nel periodo 1985-1990 (in miliardi di Ecu a prezzi e tassi di cambio correnti)

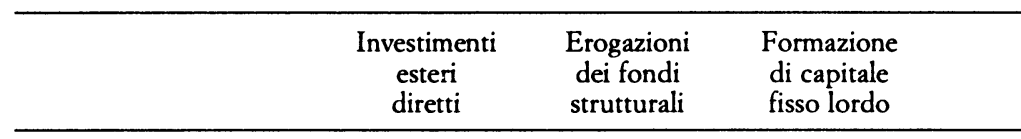

Spagna

$\begin{array}{llll}1985 & 2,50 & - & 41,87 \\ 1986 & 3,39 & 0,49 & 45,81 \\ 1987 & 3,77 & 0,68 & 52,87 \\ 1988 & 5,69 & 1,04 & 66,11 \\ 1989 & 7,21 & 1,62 & 83,41 \\ 1990 & \text { n.d. } & 2,32 & 95,42\end{array}$

Portogallo

1985

1986

1987

1988

1989

1990

Irlanda
0,34

0,23

0,37

0,70

1,42

n.d.

0,54
0,09
0,52
0,49
1,45
n.d.

0,54

0,09

0,49

1,45

1990

Grecia
0,21

0,34

0,19

0,15

0,34

n.d.

-
0,30
0,44
0,63
0,78
1,06

5,90

6,64

7,69

9,48

10,87

12,39

1986

1988

1989

1990

0,36

0,35

0,47

0,40

0,48

0,59

4,74

4,63

4,31

4,69

5,69

6,38

munitario di sostegno del 1989 ha comportato un aumento marcato degli investimenti privati di origine estera.

I dati relativi alla Spagna riportati nella tab. 2 confermano che le regioni spagnole hanno attraversato un periodo difficile a 
cavallo del 1980. I dati relativi agli investimenti e al Pil pro capite mettono in evidenza anche la notevole ripresa della seconda metà degli anni ottanta. Già nel periodo 1975-1985 alcune regioni spagnole (Extremadura e Baleari) avevano un elevato tasso di crescita del Pil pro capite. Nel corso degli ultimi quattro anni, le economie regionali di Murcia, Comunidad Valenciana e Castilla-La Mancha hanno espresso tassi di crescita medi superiori al $5 \%$.

Anche in Portogallo si sono avuti tassi di crescita significativi per la maggior parte dello stesso periodo, specie nelle regioni di Lisboa, Vale do Tejo e Centro. A partire dal 1985 anche altre tre regioni - Norte, Alentejo e Algarve - hanno espresso tassi di sviluppo superiori al tasso medio comunitario. Il Pil pro capite dal 1986 al 1990 è aumentato di 1,5 punti percentuali in più rispetto alla media comunitaria; il Pil pro capite è passato dal 52,0\% di quello medio comunitario nel 1985 al 56,3\% nel 1991 (Madureira-Pires 1992, 6) ${ }^{7}$.

La variazione percentuale annua del Pil pro capite è un indicatore non del tutto adeguato dello sviluppo economico regionale, in quanto fornisce informazioni sulle trasformazioni interne delle regioni ma non sulle trasformazioni esterne o sui confronti interregionali. Per Grecia, Spagna e Portogallo la mancanza di dati comparati a livello regionale per tutti gli anni settanta ci costringe a limitare l'analisi longitudinale dello sviluppo economico al periodo che va dal 1981 al 1990. I dati nelle tabb. 4 e 5 indicano che $i$ tre nuovi paesi membri sono stati interessati da trasformazioni anche in quest'ultimo periodo. Nel corso degli anni ottanta si è avuto un miglioramento degli indici del Pil nella maggior parte delle regioni, pur con qualche cedimento in alcune delle regioni più povere della Grecia e del Portogallo. Nel 1990 e nel 1991 le regioni portoghesi hanno cominciato a superare le regioni greche.

Se si aggregano $i$ tre stati periferici e gli altri nove paesi membri, il rapporto tra il $\mathrm{Pil}$ delle prime cinque regioni e quello delle ultime cinque passa da 7,4 nel 1981 a 6,9 nel 1989 (tab. 4). La convergenza continua per tutti gli anni ottanta, anche se - come ci si dovrebbe attendere dato che si tratta di un periodo più breve - non nella stessa misura della convergenza tra le regioni dei primi nove paesi membri nel ventennio precedente

7 Il Portogallo presentava un indice pari a 38,7 nel 1960 e a 48,9 nel 1970 (Cec 1992, appendice, 1). 
TAB. 4. Pil pro capite in Ecu nelle regioni di Grecia, Spagna e Portogallo nel periodo 1981-1989 (numeri indice con la media Cee $=100$ )

\begin{tabular}{|c|c|c|c|c|c|c|c|}
\hline & 1981 & 1984 & 1986 & 1987 & 1988 & 1989 & $\begin{array}{l}\text { Differenza } \\
1989-1981\end{array}$ \\
\hline Anat. Mak. Thraki & 44 & 52 & 42 & 41 & 42 & 44 & 0 \\
\hline Kentriki Maked. & 48 & 49 & 40 & 38 & 39 & 41 & -7 \\
\hline Kytiki Makedonia & 46 & 43 & 35 & 34 & 35 & 37 & -9 \\
\hline Thessalia & 48 & 48 & 39 & 37 & 38 & 41 & -7 \\
\hline Ipeiros & 39 & 39 & 32 & 30 & 31 & 33 & -6 \\
\hline Ionia Nisia & 44 & 46 & 38 & 36 & 37 & 40 & -4 \\
\hline Dykiti Ellada & 45 & 46 & 38 & 36 & 37 & 39 & -6 \\
\hline Sterea Ellada & 65 & 62 & 51 & 49 & 50 & 53 & -12 \\
\hline Peloponnisos & 54 & 52 & 43 & 41 & 42 & 45 & -9 \\
\hline Attiki & 52 & 54 & 44 & 42 & 44 & 40 & -12 \\
\hline Voreio Aigaio & 35 & 37 & 30 & 29 & 30 & 32 & -3 \\
\hline Notio Aigiao & 47 & 51 & 42 & 40 & 41 & 44 & -3 \\
\hline Kriti & 43 & 45 & 37 & 35 & 36 & 38 & -5 \\
\hline Galicia & 55 & 52 & 54 & 55 & 59 & 56 & +1 \\
\hline Asturias & 70 & 64 & 66 & 68 & 72 & 68 & -2 \\
\hline Cantabria & 71 & 65 & 61 & 63 & 67 & 72 & -9 \\
\hline Pais Vasco & 82 & 73 & 75 & 77 & 82 & 78 & -4 \\
\hline Navarra & 81 & 73 & 74 & 76 & 81 & 78 & -3 \\
\hline La Rioja & 81 & 77 & 76 & 78 & 83 & 78 & -3 \\
\hline Aragon & 67 & 67 & 68 & 70 & 74 & 72 & +5 \\
\hline Madrid & 74 & 69 & 72 & 73 & 78 & 74 & 0 \\
\hline Castilla y Leon & 60 & 60 & 60 & 61 & 65 & 62 & +2 \\
\hline Castilla-La Mancha & 52 & 47 & 51 & 53 & 56 & 55 & +3 \\
\hline Extremadura & 40 & 41 & 41 & 42 & 45 & 42 & +2 \\
\hline Cataluña & 73 & 69 & 71 & 73 & 77 & 75 & +2 \\
\hline Comunidad Valenciana & 64 & 60 & 63 & 65 & 69 & 67 & +3 \\
\hline Baleares & 81 & 87 & 92 & 95 & 101 & 95 & +14 \\
\hline Andalucia & 49 & 46 & 49 & 50 & 53 & 51 & +2 \\
\hline Murcia & 56 & 53 & 56 & 57 & 61 & 59 & +3 \\
\hline Norte & 28 & 25 & 25 & 25 & 26 & 25 & -3 \\
\hline Centro & 27 & 24 & 30 & 30 & 31 & 32 & +5 \\
\hline Lisboa e Vale do Tejo & 44 & 38 & 42 & 42 & 43 & 41 & -3 \\
\hline Alentejo & 28 & 25 & 27 & 28 & 28 & 29 & +1 \\
\hline Algarve & 31 & 24 & 27 & 28 & 28 & 28 & -3 \\
\hline
\end{tabular}

(vedi Leonardi 1993b). Nelle regioni più sviluppate della Comunità vi è stato un rallentamento della crescita del Pil per quanto riguarda sia le singole regioni sia i gruppi di regioni con le migliori prestazioni. Dodici delle tredici regioni greche e tre delle cinque regioni portoghesi esprimono un aumento della distanza del Pil pro capite dalla media Cee dal 1981 al 1989.

Tuttavia, questo peggioramento non interessa le due regioni portoghesi di Centro e Alentejo né le regioni spagnole coperte 
TAB. 5. Parità di potere d'acquisto pro capite nelle regioni di Grecia, Spagna e Portogallo nel periodo 1981-1989 (numeri indice con la media Cee $=100$ )

\begin{tabular}{lrrrrrrr}
\hline & & & & & & & Differenza \\
& 1981 & 1984 & 1986 & 1987 & 1988 & 1989 & $1989-1981$ \\
\hline Anat. Mak. Thraki & 55 & 63 & 63 & 61 & 61 & 62 & +7 \\
Kentriki Maked. & 61 & 59 & 59 & 57 & 58 & 59 & -2 \\
Kytiki Makedonia & 59 & 52 & 52 & 51 & 51 & 55 & -4 \\
Thessalia & 61 & 58 & 58 & 56 & 56 & 57 & -4 \\
Ipeiros & 49 & 47 & 47 & 46 & 46 & 44 & -5 \\
Ionia Nisia & 55 & 56 & 56 & 55 & 55 & 55 & 0 \\
Dykiti Ellada & 57 & 56 & 56 & 54 & 55 & 52 & -5 \\
Sterea Ellada & 83 & 76 & 75 & 73 & 74 & 76 & -7 \\
Peloponnisos & 68 & 63 & 63 & 61 & 62 & 63 & -5 \\
Attiki & 66 & 66 & 65 & 64 & 64 & 62 & -4 \\
Voreio Aigaio & 44 & 45 & 45 & 43 & 44 & 44 & 0 \\
Notio Aigiao & 60 & 62 & 62 & 61 & 61 & 59 & -1 \\
Kriti & 54 & 54 & 54 & 53 & 53 & 58 & +4 \\
& & & & & & & \\
Galicia & 85 & 68 & 68 & 70 & 71 & 65 & -3 \\
Asturias & 87 & 84 & 84 & 86 & 87 & 83 & -4 \\
Cantabria & 88 & 85 & 78 & 80 & 81 & 81 & -7 \\
Pais Vasco & 102 & 96 & 96 & 98 & 99 & 103 & +1 \\
Navarra & 101 & 96 & 95 & 97 & 99 & 107 & +6 \\
La Rioja & 101 & 100 & 97 & 99 & 101 & 96 & -5 \\
Aragon & 83 & 88 & 87 & 89 & 90 & 92 & +9 \\
Madrid & 91 & 91 & 91 & 94 & 95 & 104 & +13 \\
Castilla y Leon & 74 & 78 & 76 & 78 & 79 & 78 & +4 \\
Castilla-La Mancha & 64 & 62 & 65 & 67 & 68 & 72 & +8 \\
Extremadura & 49 & 54 & 53 & 54 & 55 & 54 & +5 \\
Cataluña & 90 & 91 & 90 & 93 & 94 & 104 & +14 \\
Comunidad Valenciana & 79 & 79 & 81 & 83 & 84 & 86 & +7 \\
Baleares & 100 & 113 & 117 & 121 & 122 & 114 & +14 \\
Andalucia & 60 & 60 & 62 & 63 & 64 & 65 & +5 \\
Murcia & 69 & 69 & 71 & 73 & 73 & 81 & +12 \\
Ceuta y Melilla & 54 & 52 & 57 & 59 & 59 & 70 & +16 \\
Canarias & 67 & 71 & 78 & 79 & 80 & 86 & +19 \\
& 50 & 48 & 45 & 46 & 46 & 52 & +2 \\
Norte & 48 & 45 & 54 & 56 & 56 & 50 & +2 \\
Centro & 79 & 74 & 75 & 77 & 77 & 77 & -2 \\
Lisboa e Vale do Tejo & 50 & 48 & 50 & 51 & 51 & 55 & +5 \\
Alentejo & 55 & 46 & 50 & 51 & 51 & 55 & 0 \\
Algarve & & & & & & & \\
\hline
\end{tabular}

dall'«Obiettivo $1 »$ dei fondi strutturali (tale obiettivo è la promozione dello sviluppo delle regioni sottosviluppate).

In Spagna, le regioni di Cantabria, Pais Vasco e Navarra, che non rientrano nell'ambito dell'Obiettivo 1 , subiscono un calo; ma tutte le altre regioni esprimono una crescita. Anzi, l'omogeneità della Spagna è aumentata di molto dal 1981 al 
1990; i dislivelli interni sono diminuiti. Questo andamento implica che sono diminuite anche le differenze nei dislivelli tra la Comunità e la Spagna. In Spagna e in Portogallo si ha una tendenza più marcatamente positiva per quanto riguarda l'indice Pps (purchasing power standard - parità di potere d'acquisto ovvero la capacità di spesa o, per estensione, il tenore di vita) nel periodo che va dal 1981 al $1989^{8}$.

Vale la pena notare che in sei regioni spagnole vi è una forte crescita del Pil pro capite e, in particolare, dell'indice Pps (vedi tabb. 4 e 5). Le regioni per le quali l'indice Pps cresce di più sono le «capitali» turistiche - Baleari e Canarie - le aree metropolitane di Madrid e Catalogna, il territorio di Ceuta y Melilla e la regione periferica di Murcia'. L'economia di ciascuna di queste regioni ha una forte componente turistica.

Pare che in Spagna il turismo stia diventando sempre più importante nella stimolazione dello sviluppo delle economie regionali precedentemente sottosviluppate. Forse il turismo funge da valvola di sicurezza per il declino della produzione industriale e per la crescente disoccupazione a metà degli anni ot$\operatorname{tanta}^{10}$. Forse anche la Grecia farebbe bene ad avvalersi dello sviluppo del turismo come strategia di punta nella promozione dello sviluppo economico delle aree periferiche.

Un secondo aspetto da tener presente quando si confrontano le regioni portoghesi e greche con quelle spagnole è che queste ultime per promuovere lo sviluppo hanno a disposizione una serie di leve che invece non sono disponibili in Portogallo e Grecia. Le regioni spagnole possiedono l'«infrastruttura istituzionale» che occorre per poter intraprendere progetti di selfhelp o mettere alla prova forme alternative di sviluppo economico. L'autonomia politica e amministrativa permette alle regioni di sperimentare politiche senza dover aspettare che il go-

8 Un andamento simile si riscontra in Portogallo, dove la regione del Centro passa, in termini di capacità di spesa, dall'ultima posizione nel 1981 alla seconda nel 1989, scavalcando il Norte e l'Algarve.

9 La mancanza di informazioni sul Pil delle isole Canarie fa sì che l'analisi sia incompleta, ma l'entità dell'aumento del Pps dal 1981 al 1989 indica che sarebbe ragionevole aspettarsi un andamento parallelo per quanto riguarda il Pil. I dati sulle regioni spagnole suggeriscono che sia in atto un forte sviluppo della coesione interna nell'ambito dell'integrazione della Spagna nella Comunità europea. In Portogallo risulta essere in atto un fenomeno analogo.

${ }_{10} \mathrm{Da}$ una mia recente indagine sui flussi e sulle entrate di natura turistica nel Mediterraneo (Eiu 1992) risulta che il volume di turisti è diminuito leggermente dal 1989 al 1990 , contro un aumento delle entrate lorde del $25 \%$. 
verno nazionale escogiti soluzioni adeguate. Le regioni greche e portoghesi non hanno alcun accesso a strumenti alternativi di questo genere; quindi è più probabile che debbano partecipare a progetti formulati a livello nazionale, che richiedono necessariamente più tempo perché i loro effetti si facciano sentire a livello regionale. La paralisi che caratterizza la struttura amministrativa greca costituisce un elemento di inquietudine costante per coloro che attuano progetti di sviluppo locale; questa situazione non potrà essere riformata in modo significativo finché le strutture decisionali e amministrative non verranno ristrutturate e decentrate.

In terzo luogo va ribadito che, benché lo sviluppo di queste regioni sia stato preso in esame per un periodo di nove anni, i rispettivi stati nazionali non hanno fatto parte della Comunità per lo stesso periodo: Portogallo e Spagna hanno aderito solo nel 1986. Una delle nostre ipotesi di lavoro è che l'integrazione di per sé funga da stimolo positivo alle economie regionali periferiche e dia conto di una quota consistente della convergenza.

Gli indici complessivi di Spagna e Portogallo manifestano una tendenza negativa fino al 1986 per poi riprendersi in seguito all'adesione alla Comunità. Di conseguenza, la maggior parte dei dati riportati nelle tabelle e relativi a questi due paesi attiene a trasformazioni nelle economie regionali intervenute prima dell'ingresso nella Comunità e a prescindere da qualunque specifico programma comunitario. Pertanto, questi dati rispecchiano i cambiamenti del grado di coesione economica e sociale prima che l'integrazione europea e la partecipazione a programmi comunitari mirati a promuovere lo sviluppo avessero potuto avere un qualche effetto. Una volta pienamente operanti, questi programmi hanno avuto un impatto notevole sulle attività economiche dei tre nuovi paesi membri.

Gli aiuti comunitari, sotto forma di fondi strutturali nell'ambito dei programmi del quadro comunitario di sostegno, hanno contribuito molto ai livelli di investimento e al Pil complessivo in questi tre paesi: $3,5 \%$ del $\mathrm{Pil}$ in Portogallo, $1,2 \%$ in Spagna e 2,9\% in Grecia. Si tratta di stimoli positivi, cui vanno aggiunti i contributi nazionali pari al $3,1 \%, 0,8 \%$ e $2,2 \%$, rispettivamente, in Portogallo, Spagna e Grecia (Nanetti 1992).

Nonostante l'effetto positivo di questo e di altri programmi di spesa comunitari, le statistiche ufficiali dimostrano che la situazione economica non è migliorata in Grecia. Se si vuole dare 
credito alle statistiche ufficiali, il culmine della convergenza tra la Grecia e le restanti economie nazionali della Comunità si è avuto nel 1978, quando la Grecia stava ancora negoziando le modalità della propria adesione. In quell'anno il Pil pro capite raggiunse il 58,6\% della media Cee e da allora è sempre diminuito. La Grecia è entrata a far parte della Comunità europea nel 1981 e ha beneficiato di alcuni dei maggiori programmi di spesa. Come mai i flussi di investimenti dalla Comunità non hanno avuto alcun effetto? Come mai la Grecia è l'unico paese che smentisce sistematicamente le teorie della convergenza?

Per ottenere la risposta si può procedere in molte direzioni. In primo luogo, la Grecia aderì alla Comunità all'inizio di una recessione che ha coinvolto l'Europa intera. La debole economia greca, pertanto, non ha potuto approfittare di un mercato europeo in espansione né di una grande disponibilità di risorse.

In secondo luogo, la capacità della Grecia di sfruttare appieno gli aiuti comunitari per lo sviluppo ha sempre lasciato a desiderare. Quando furono introdotti i programmi integrati mediterranei, i quali hanno comportato un esborso di 3,3 miliardi di Ecu a favore della Grecia nel corso di sette anni, Francia e Italia riuscirono a trarre vantaggio da questi programmi più della Grecia. Anche in questo caso la scadente infrastruttura istituzionale a livello subnazionale ha rallentato lo sviluppo economico e l'attuazione dei programmi comunitari, anche se la Grecia ha un bisogno disperato di un rapido susseguirsi di programmi per mettere in moto lo sviluppo.

In terzo luogo, in Grecia la base imprenditoriale non è sufficientemente organizzata per riuscire a collegare in modo efficace tra loro singoli imprenditori, associazioni, gruppi settoriali e programmi comunitari a favore degli investimenti. L'accesso ai programmi comunitari viene ancora regolato secondo una struttura clientelare, non per affrontare l'obiettivo dello sviluppo economico. Ciò è particolarmente evidente nell'incapacità della Grecia di accedere ai prestiti della Banca europea per gli investimenti (Bei). L'ammontare dei prestiti ricevuti dalla Grecia è molto inferiore a quello dei prestiti concessi ad altri paesi sottosviluppati come Spagna e Portogallo, e i tipi di progetti finanziati dalla Bei sono molto più orientati all'infrastruttura che non a specifici settori, ovvero, nelle parole della stessa Bei, a stimolare «la competitività industriale e l'integrazione europea» (Bei 1991, 80-101). E necessario compiere sforzi particolari in Grecia al fine di favorire l'organizzazione e la formazione di reti 
di comunicazione tra gli imprenditori e di incoraggiare l'integrazione degli interessi economici greci nella struttura delle opportunità offerta dal mercato unico.

In quarto luogo, come si è già accennato, le spese relative al quadro comunitario di sostegno non sono state accompagnate $\mathrm{da}$ investimenti stranieri: gli investimenti nazionali e comunitari non hanno avuto l'effetto di richiamare investimenti privati da altri paesi. Il capitale straniero è rimasto lontano dalla Grecia. La colpa può essere attribuita in parte alla mancanza di trasparenza amministrativa e all'esclusione della moneta greca dal sistema monetario europeo, ma la restante parte è attribuibile all'assenza in periferia di programmi di investimento orientati al futuro.

\section{I fattori che determinano lo sviluppo dell'Europa meridionale}

Dopo aver ripercorso i cambiamenti nei livelli di produttività e di benessere, è necessario individuare alcune variabili esplicative. La ricerca di variabili indipendenti trova un limite nella disponibilità di dati che siano articolati a livello regionale. Fino ad ora abbiamo potuto analizzare soltanto quattro variabili per accertare la loro capacità esplicativa in relazione alla variazione di entrambe le variabili dipendenti. Per una quinta variabile l'entità degli investimenti di capitale - i dati articolati a livello regionale non sono sufficienti per alcuni paesi e pertanto non possono essere impiegati in un'analisi longitudinale.

$\mathrm{Dal}$ controllo delle quattro variabili indipendenti nei confronti degli indici Pil e Pps, emerge quanto segue:

A. La distanza costituisce la variabile con maggiore capacità predittiva delle variazioni nel Pil e nelle Pps. Ne consegue che le regioni lontane dal centro si stanno trasformando più rapidamente di quelle interne o vicine al centro, il che conforta la tesi dell'ascesa periferica. Tuttavia, la distanza non è una variabile esplicativa pienamente soddisfacente, in quanto non ci indica quali fattori di sviluppo (investimenti, livelli retributivi, innovazioni tecnologiche, ecc.) alimentino le variazioni nel Pil e nelle Pps. Sappiamo solo che le trasformazioni sono in atto e dove esse avvengono, ma non abbiamo indizi precisi sul perché avvengono né su come l'integrazione agisce sulle economie regionali. Per rispondere a questi interrogativi è necessario disporre di una base-dati più completa e articolata di quella attualmente a disposizione della Comunità. 
B. Il livello di industrializzazione nel 1970 è una variabile meno efficace nella previsione delle variazioni del Pil e delle Pps. La percentuale di forza lavoro impiegata nell'industria è correlata con gli indici, ma non in misura significativa. Ne consegue che il ciclo industriale può anche svolgere un ruolo nella spiegazione delle trasformazioni, ma non può certo spiegarle per intero. Forse ciò è dovuto al fatto che negli anni settanta e ottanta i governi nazionali e regionali sono ricorsi a diversi livelli di investimento mentre ristrutturavano le loro industrie malandate. Da un'indagine della Comunità sulle sovvenzioni statali all'economia (Cec 1990) si evince che nel periodo che va dal 1981 al 1988 gli aiuti al settore manifatturiero, esclusi la cantieristica navale e le acciaierie, sono cresciuti in modo marcato in Italia e in Germania e diminuiti nel Regno Unito e in Irlanda. Grazie agli aiuti forniti dai governi nazionali e regionali, il ciclo di ristrutturazione industriale è stato forse più breve in Italia e in Germania che non nel Regno Unito, dove il declino industriale è stato giustificato nell'ambito di una politica industriale selettiva che mirava ad eliminare le imprese non competitive e a ridurre i sussidi governativi. Un altro motivo sta nella crescente terziarizzazione dell'economia nei paesi sia sviluppati sia meno sviluppati. In questo modo la distinzione tra economie centrali e periferiche è diventata meno nitida della classica distinzione tra occupazione industriale e occupazione nei servizi.

C. Il livello di disoccupazione quale variabile esplicativa funziona un po' meglio del livello di industrializzazione. Questa variabile è correlata negativamente sia con il Pil sia con le Pps, ed entrambe le correlazioni sono significative; ma la correlazione è più debole di quella prodotta dalla variabile di distanza. La diminuzione dei tassi di disoccupazione è un importante indicatore di produttività crescente e di benessere, ma non è di per sé sufficiente. L'avvento del welfare state, l'espansione delle burocrazie pubbliche e l'erogazione di indennità di disoccupazione hanno ridotto gli effetti negativi della disoccupazione sul benessere ma anche la mobilità della forza lavoro - cioè l'incentivazione a cercare lavoro laddove vi sono delle penurie di forza lavoro - come accadde negli anni cinquanta e sessanta. La migrazione della forza lavoro non basta più a spiegare la convergenza.

Elevati livelli di disoccupazione, infatti, costituiscono un corollario dell'occupazione stagionale: laddove l'occupazione stagionale rappresenta una componente importante dell'economia 
regionale - ad esempio nel settore del turismo - vi è anche un'elevata incidenza della disoccupazione. Dietro un'elevata disoccupazione in aree con economie diversificate e con un forte settore dei servizi, possono nascondersi forme di occupazione stagionale. In queste aree i livelli di produttività e di benessere possono anche aumentare, ma $\mathrm{i}$ tassi di disoccupazione rimarranno molto elevati.

D. Un'ulteriore variabile esplicativa riguarda il livello di spesa della Comunità europea nelle regioni, in forme che vanno dagli aiuti del Fondo di sviluppo regionale europeo ai prestiti della Banca europea per gli investimenti. L'analisi dimostra che nel corso del tempo vi è un aumento della correlazione $(p<0,05)$ tra l'insieme dei contributi comunitari alle singole regioni e le variazioni negli indici regionali di Pil e di Pps. La riforma e il raddoppio dei fondi strutturali nel 1988 e la successiva decisione di fare altrettanto per il periodo che va dal 1994 al 1999 indicano che è stato rafforzato ed è destinato ad espandersi ancora in futuro l'effetto dei contributi comunitari sull'accelerazione dello sviluppo e sull'aumento del benessere nelle regioni meno sviluppate. In altri termini, dai dati si evince che l'aumento degli aiuti comunitari serve davvero a conseguire la coesione economica e sociale, e probabilmente è uno dei fattori più importanti per ottenere questa coesione in maniera ancora più rapida.

Questa aspettativa è riferibile in modo particolare ai tre stati membri che rientrano nell'ambito dell'Obiettivo 1: Irlanda, Grecia e Portogallo. Se si considera che alla fine del $1993 \mathrm{gli}$ aiuti dei fondi strutturali incideranno per almeno il $3 \%$ circa del Pil, non sorprende che la Comunità europea venga vista come un partner importante per lo sviluppo economico degli stati membri periferici. Nel caso specifico del Portogallo, nel corso del periodo quinquennale di durata del quadro comunitario di sostegno, i finanziamenti comunitari ammontavano al $30 \%$ degli investimenti totali, al $60 \%$ del piano annuo di investimenti del governo centrale e al $40 \%$ degli investimenti delle amministrazioni comunali (Madureira-Pires 1992).

E. Il tasso di investimento viene spesso usato per dar conto delle differenze nei tassi di sviluppo delle economie regionali. Tuttavia, la natura frammentaria dei dati non ci permette di usare questa variabile per spiegare le variazioni di Pil e di Pps. Mancano spesso alcuni dati relativi a Francia, Belgio e Regno Unito e, in misura minore, a Irlanda e Danimarca. Così non possiamo accertare se $\mathrm{i}$ tassi di investimento siano o meno cor- 
relati con i nostri indici. Alcuni dati riferiti al livello nazionale, tuttavia, indicano che una combinazione di investimenti provenienti dai fondi strutturali e di aumenti negli investimenti interni e esteri fa sì che l'effetto globale delle strategie di investimento promosse dalla Comunità sia ben al di sopra della soglia del 5\% del Pil, indicata dagli economisti come soglia minima se si vuole che le economie regionali si sviluppino più velocemente di quelle centrali.

Le quattro variabili indipendenti appena illustrate offrono indicazioni preziose circa gli elementi potenzialmente connessi alle variazioni delle variabili dipendenti. La distanza dal centro ha la migliore capacità predittiva rispetto alle variabili dipendenti. Anche i tassi di spesa comunitaria esprimono una relazione sempre più forte con i tassi di sviluppo economico. Il tasso di disoccupazione e la fase di industrializzazione, infine, manifestano una minore capacità esplicativa. La debolezza di queste ultime due variabili è forse dovuta alla trasformazione della struttura delle economie industrializzate (in particolare, il passaggio dalle grandi aziende industriali e dalle economie di scala ai cicli produttivi più flessibili basati su piccole unità di produzione e servizi tecnologici misti) e alle caratteristiche peculiari di quelle economie che dipendono in misura notevole dalle attività e dalle occupazioni stagionali. Presi insieme, questi fattori spiegano una parte della convergenza, anche se non tutta, che ha interessato gli stati periferici dell'Europa meridionale negli anni ottanta.

\section{Il Mezzogiorno italiano e il modello di convergenza}

Il Mezzogiorno italiano presenta una serie di caratteristiche simili a quelle di altre regioni distribuite lungo il lato settentrionale del Mediterraneo. Prima dell'adesione di Grecia, Portogallo e Spagna, esso aveva i più bassi livelli di sviluppo tra quelli registrati tra le regioni della Comunità; e anche la sua offerta di infrastrutture sociali ed economiche era tra le più carenti. Nel 1950 il livello di sviluppo dell'Italia meridionale era circa un terzo della media dei primi nove membri della Comunità. La sua risorsa principale, un surplus di forza lavoro, veniva liberamente esportata nelle regioni italiane del Nord, in particolare nel Triangolo industriale, e nei paesi industrializzati appartenenti alla Comunità europea. 
TAB. 6. Variazioni nel Pil pro capite nelle regioni meridionali italiane nel periodo 1950 1990 (numeri indice con la media dei primi nove membri Cee $=100$ )

\begin{tabular}{lccccccc}
\hline & \multicolumn{3}{c}{ In dollari } & \multicolumn{5}{c}{ In Ecu } \\
\hline & 1950 & 1960 & 1970 & 1970 & 1981 & 1986 & 1990 \\
Campania & 42 & 42 & 48 & 55 & 55 & 60 & 63 \\
Abruzzo & 41 & 41 & 54 & 56 & 65 & 78 & 84 \\
Molise & 34 & 35 & 44 & 46 & 58 & 69 & 75 \\
Puglia & 40 & 34 & 50 & 55 & 56 & 63 & 69 \\
Basilicata & 35 & 29 & 46 & 46 & 56 & 56 & 59 \\
Calabria & 34 & 30 & 41 & 42 & 49 & 51 & 54 \\
Sardegna & 46 & 43 & 55 & 54 & 56 & 67 & 71 \\
Sicilia & 41 & 37 & 52 & 54 & 54 & 51 & 65 \\
\hline
\end{tabular}

Fonte: Molle et al. (1980) per il periodo 1950-1970; Leonardi (1993a) per il periodo 1970-1990.

Nella tab. 6 sono riportati alcuni dati relativi al Pil pro capite, espresso in dollari (1950-1970) e in Ecu (1970-1990), delle otto regioni meridionali italiane e delle altre regioni dei primi stati membri della Comunità. Nel 1950, tre regioni - Molise, Basilicata e Calabria - si trovavano agli ultimi posti, mentre la situazione nelle altre cinque era leggermente migliore. La Sardegna e la Campania erano le regioni meridionali più forti; la Sicilia e la Puglia occupavano posizioni intermedie. Nel 1960 la situazione complessiva delle otto regioni, dissanguate dall'emigrazione e dalla fuga dei capitali, era peggiorata. Solo il Molise, l'Abruzzo e la Campania erano riuscite a mantenere le loro posizioni, mentre le altre avevano subìto uno sviluppo negativo rispetto al decennio precedente.

Nel corso degli anni sessanta il livello di sviluppo nell'Italia meridionale subì una forte inversione di tendenza. Nel 1970 molte regioni presentavano livelli di reddito pari alla metà della media Cee. Nel corso degli ultimi due decenni le regioni del Mezzogiorno hanno continuato a migliorare i propri livelli di benessere in termini sia relativi sia assoluti, sicché nel 1992 alcune regioni - Abruzzo, Molise e Sardegna - hanno raggiunto un livello di sviluppo pari al $75 \%$ della media Cee. Per la seconda fase del quadro comunitario di sostegno, prevista per il periodo 1994-1999, l'Abruzzo non sarà più riconosciuto come una regione coperta dall'Obiettivo 1 . Il Molise e la Sardegna, invece, benché superino la soglia del $75 \%$, non saranno esclusi 
da questa seconda fase in quanto la soglia di eleggibilità di fatto è stata elevata dal 75 all' $80 \%$ della media $\mathrm{Cee}^{11}$.

Il livello del Pil pro capite e i tassi di sviluppo nel Mezzogiorno indicano in modo netto che esso non costituisce un'area omogenea. Alcune regioni - tra cui spiccano quelle piccole di Abruzzo, Molise e Basilicata - si stanno sviluppando più rapidamente di altre. Le economie regionali di Campania, Sicilia, Puglia e Calabria, invece, non hanno ottenuto buoni risultati, nonostante i loro vantaggi di partenza riguardo al livello di sviluppo agricolo, alla presenza di grandi aree metropolitane e a consistenti investimenti industriali pubblici. Se a questi dati si aggiungono i risultati di uno studio del rendimento istituzionale delle venti regioni italiane lungo un arco di vent'anni (Putnam 1993), si evince che le più grandi regioni dell'Italia meridionale si distinguono per le loro prestazioni scadenti. Per quale motivo le grandi regioni del Mezzogiorno hanno conseguito risultati deludenti rispetto alle regioni piccole?

La risposta va cercata in diversi ambiti. Dal punto di vista della cultura politica, si registra una carenza di cultura civica nelle popolazioni del Meridione e una prevalenza di orientamenti clientelari negli importanti processi di formazione dei legami tra:

- la classe politica e i cittadini nell'elaborazione delle domande politiche e nell'attuazione delle decisioni politiche;

- gli operatori economici e le autorità pubbliche nella distribuzione delle risorse pubbliche e nella regolazione delle attività di mercato;

- le organizzazioni criminali e le istituzioni politiche nell'affrontare le attività illecite e l'abuso dell'autorità pubblica.

La prevalenza di queste tre caratteristiche tende a disincentivare lo sviluppo di un senso di comunità e le conseguenti forme di economia diffusa che hanno contribuito ad alimentare la crescita economica in altre parti d'Italia. Inoltre, la dipendenza dei mercati politici regionali da strutture di potere legate ai partiti di governo e alle élites nazionali dominanti, in grado di accedere a risorse pubbliche di notevole entità, ha fatto sì che si

11 Va ricordato che la base per il calcolo degli indici nella tab. 6 si riferisce ai primi nove paesi membri della Cee e non a tutti gli attuali dodici membri. Il Molise e la Sardegna vengono ancora coperti dall'Obiettivo 1 grazie a negoziazioni politiche tra gli stati membri. Il nuovo compromesso sui criteri di inclusione ha permesso anche a Irlanda del Nord, Corsica e Valencia di continuare a beneficiare degli aiuti destinati alle regioni che fanno capo all'Obiettivo 1 . 
privilegiassero iniziative nazionali piuttosto che regionali o locali. La prevalenza di strutture di rapporti basate sul centro non ha incoraggiato la formazione di un forte senso di autonomia regionale e quindi non ha consentito la mobilitazione di forme endogene di sviluppo basate su strategie originate dal basso.

Dal punto di vista economico, lo sviluppo dell'iniziativa privata al Sud è stato sopraffatto da una forte prevalenza di interventi pubblici nel settore produttivo per mezzo di grandi imprese pubbliche, dalle sovvenzioni pubbliche alle attività economiche, da un controllo centralizzato sulla distribuzione delle risorse mediante programmi speciali per il Sud e dall'adozione di politiche di sviluppo ostili alla libera interazione di forze di mercato. Sin dall'inizio della politica italiana per lo sviluppo del Meridione - esplicata mediante la creazione della Cassa per il Mezzogiorno nel 1950 e, successivamente, con l'Agenzia per la promozione dello sviluppo del Mezzogiorno - la stessa esistenza di forze di mercato nell'economia meridionale è stata messa in forse.

Alcuni sondaggi recenti evidenziano come l'elevato livello di intervento pubblico e la facilità di accesso a fondi pubblici grazie all'intermediazione politica abbiano generato un elevato grado di risentimento tra $\mathrm{i}$ piccoli e medi imprenditori privati. Questi ultimi lamentano la difficoltà a far fronte alla concorrenza di imprese pubbliche o di aziende private che si avvalgono di consistenti sussidi nazionali - operatori che possono permettersi di non tener conto delle regole della concorrenza, in quanto consapevoli che le eventuali perdite verranno compensate $\mathrm{da}$ sussidi pubblici (vedi Leonardi 1991).

Di conseguenza, l'inesistenza al Sud di un vero mercato degli investimenti e della produzione ha ostacolato l'emergere di forme collettive di imprenditorialità (ad esempio, cooperative di produttori o di istituzioni politiche locali), le quali avrebbero potuto stimolare politiche di investimento e strategie di sviluppo alternative. La sostituzione dell'intervento statale a un mercato concorrenziale ha portato a un notevole declino della concorrenzialità delle regioni meridionali in relazione alla capacità di esportare i loro manufatti e la loro produzione agricola ai mercati italiani ed europei.

Se si confrontano i dati relativi alle regioni dell'Italia meridionale con quelli delle altre regioni periferiche dell'Europa del Sud, si giunge alla conclusione che, a meno che non si spezzi la stretta mortale della classe politica italiana sull'attività economi- 
ca del Mezzogiorno e non vengano reintrodotti criteri di mercato nelle decisioni di investimento e di produzione, l'area rischia di soccombere alle regioni spagnole e portoghesi in via di sviluppo. Le autonomie locali non hanno avuto la possibilità di avvalersi pienamente dei fondi per lo sviluppo regionali, nazionali o comunitari. Anzi, nel caso dei programmi integrati mediterranei, Sicilia, Campania e Calabria hanno visto un'ampia quota delle risorse loro già assegnate essere dirottata a favore della Francia a causa della loro incapacità di spendere il denaro entro i termini e secondo le modalità indicate dalla Comunità. E probabile che $\mathrm{i}$ fondi loro destinati nell'ambito del quadro comunitario di sostegno facciano una fine analoga.

L'incapacità delle regioni italiane meridionali di adempiere ai requisiti minimi di amministrazione e di pianificazione stabiliti dalla Comunità e accettati dalle regioni stesse mette in forse il futuro dei progetti di sviluppo regionale per il Mezzogiorno. Siccome i fondi per lo sviluppo regionale sono sempre più legati alla cooperazione e all'autorizzazione da parte della Comunità, è molto importante per le regioni del Mezzogiorno cambiare non solo le proprie politiche economiche ma anche, e principalmente, il modo di gestire i fondi per lo sviluppo. I contatti politici o partitici a livello nazionale non sono più sufficienti a garantire che $\mathrm{i}$ fondi arriveranno in modo automatico e incontrollato a una specifica regione. Ora servono un maggiore grado di iniziativa e di autonomia politica regionale nella gestione dei fondi e una capacità collaudata di recepire le esigenze economiche e sociali della regione. L'Italia non può più permettersi di avere quasi una metà del paese che agisce secondo principi economici e politici che confliggono con quelli che prevalgono nel resto del paese e della Comunità. I tentativi di eliminare il disavanzo pubblico e garantire chiarezza e trasparenza nella spesa pubblica faranno venire meno le fonti nazionali di finanziamento alle regioni, le quali, in assenza delle trasformazioni summenzionate, subiranno un'ulteriore stagnazione e declino.

\section{Conclusioni}

Le conclusioni che si possono trarre dalla precedente analisi della periferia dell'Europa meridionale sono evidenti. Nel corso degli ultimi due decenni vi è stato un miglioramento complessivo nella coesione economica e sociale nella Comunità europea. 
Sono aumentati i livelli di produttività e di benessere sociale e si è ridotto il divario tra le regioni più ricche e quelle più povere. Le aspettative iscritte nel preambolo del Trattato del 1957 e messe in evidenze nell'Atto unico europeo e nel Trattato di Maastricht sono state, in una certa misura, realizzate dall'integrazione economica. È probabile che l'ulteriore integrazione che risulterà dal consolidamento del mercato unico e dell'unione monetaria europea porterà a un miglioramento delle prospettive delle regioni più deboli.

Questo sorprendente risultato è dovuto ad alcuni processi economici molto semplici. Le implicazioni politiche, a loro volta, sono evidenti e univoche. La creazione del mercato unico e dell'unione monetaria europea tenderà a ridurre le differenze tra centro e periferia per quanto riguarda la portata e la rapidità con cui gli choc economici si diffondono nella Comunità. $\mathrm{Di}$ conseguenza, nell'ambito del processo di integrazione, la Comunità dovrà accrescere la sua capacità di usare i poteri di tassazione e di trasferimento economico per attutire i colpi e attenuare i loro effetti negativi sulle economie periferiche locali; ma l'obiettivo dell'integrazione economica non va considerato antitetico allo sviluppo nazionale o regionale. Gli aiuti dei fondi strutturali non costituiscono una forma di compensazione per la partecipazione al mercato unico o all'unione monetaria europea, bensì sono strumenti per stimolare o persino accelerare la crescita nel contesto delle opportunità offerte dall'integrazione europea.

Di conseguenza, il motivo per cui si sono raddoppiati gli stanziamenti dei fondi strutturali e si è creato il Fondo di coesione dovrebbe essere l'accelerazione dello sviluppo nelle aree periferiche $e$, più in generale, nel medio periodo, la riduzione a dimensioni accettabili del divario tra regioni sviluppate e sottosviluppate. La definizione di «dimensioni accettabili» va ancora dibattuta e concordata nell'ambito della Comunità, ma è ormai evidente che la realizzazione della coesione non è più un obiettivo astratto e impossibile.

Nel rispondere ai quesiti posti in precedenza circa gli effetti economici dell'integrazione europea, abbiamo visto che:

a) Nella periferia sono aumentati i tassi di crescita.

b) Le medie e piccole imprese hanno continuato a svilupparsi.

c) Le grandi e inefficienti industrie manifatturiere hanno avuto difficoltà nella periferia ma anche al centro. L'integrazio- 
ne dei mercati ha minato alla base le grandi aziende monopolistiche pubbliche e private che in precedenza venivano sovvenzionate dai governi nazionali per motivi politici, economici e strategico-militari.

d) Sono cessate le migrazioni della forza lavoro basate sullo scambio di popolazioni all'interno della Comunità. Ora il problema di maggiore rilievo è l'immigrazione da paesi extra-comunitari, la quale va affrontata in termini non solo economici ma anche politici e amministrativi.

e) $\mathrm{Vi}$ è stata una convergenza tra le economie dei paesi e delle regioni della Comunità. La coesione socio-economica è una realtà, non più soltanto un obiettivo formale dell'integrazione europea.

Oltre alla realtà economica, l'integrazione europea ha trasformato le basi politiche e amministrative e le strategie di sviluppo degli stati nazionali e delle regioni. L'analisi comparata dimostra che la concorrenza tra le regioni comunitarie si è trasformata con l'emergere di strategie di sviluppo alternative, le quali si incentrano non solo sulla tradizionale infrastruttura economica ma anche su quella istituzionale e sociale. I governi regionali e locali, assieme alle autorità semi-pubbliche, vanno considerati come partner dello sviluppo e come anelli-chiave della catena economica che promuove la crescita economica nelle aree sia centrali sia periferiche. I confronti tra i modelli di sviluppo mettono in evidenza come, a bassi livelli di crescita e di maturità economica, i sistemi statali centralizzati siano in grado di contribuire in modo significativo alla realizzazione di economie di scala e all'impiego efficiente delle risorse. Tuttavia, quando le economie raggiungono le fasi mature dello sviluppo, le strutture amministrative centralizzate tendono a soffocare l'iniziativa locale e la ricerca di nicchie di mercato da riservare alle economie locali. In altre parole, gli attuali sistemi centralizzati di Portogallo, Irlanda e Grecia sono forse necessari per il decollo dell'economia; ma servono forme di governo decentrate per mantenere livelli maturi di rendimento economico.

Da queste tendenze si evince che gli stati nazionali dell'Europa meridionale dovranno intraprendere riforme istituzionali che prevedano la partecipazione di gruppi e di interessi locali nei processi decisionali e di attuazione in ambito socio-economico, al fine di conservare gli attuali livelli di sviluppo. In caso contrario, questi paesi rischiano una nuova ondata di emarginazione. Di conseguenza, è necessario creare amministrazioni re- 
gionali e rafforzare i poteri delle amministrazioni locali al fine sia di promuovere buone prestazioni da parte dell'economia, sia di rinsaldare il sistema democratico a livello locale mediante la concessione ai cittadini del potere di partecipare alla determinazione degli esiti locali. Istituzioni democratiche potenti e autonome non costituiscono un lusso per i regimi democratici consolidati, bensì una scuola per i principi democratici e un laboratorio sperimentale per la formulazione di politiche economiche e sociali orientate alle nicchie e capaci di recepire le esigenze delle aree locali e le loro potenzialità di sviluppo socioeconomico.

Il decentramento comporta vantaggi relativi alla promozione di uno sviluppo economico endogeno che sono ben maggiori degli svantaggi determinati dalle spese aggiuntive relative alla creazione di un nuovo livello amministrativo o all'assunzione di altro personale amministrativo a livello regionale. Pertanto, la creazione di valide forme di sistemi politico-amministrativi subnazionali, autonomi, ad elezione diretta potrebbe costituire un ulteriore indicatore della crescente convergenza delle pratiche politiche e amministrative nella Comunità e, più in generale, della necessità di accrescere la capacità delle economie regionali di rispondere alle forze di mercato e alla congiuntura economica internazionale. Nel contesto della dinamica dell'integrazione europea e dell'importanza attribuita alla trasparenza politica e all'efficacia amministrativa, gli stati e le regioni, sia del centro che della periferia, stanno diventando sempre più simili, non diversi, tra loro.

L'integrazione europea ha avuto implicazioni profonde anche per le strutture e le pratiche politiche degli stati nazionali e delle loro componenti. Ciò è particolarmente vero per quanto attiene alla periferia. Abbiamo affermato che gli stati periferici hanno guadagnato moltissimo in termini di prestigio politico e di potere effettivo. L'integrazione europea ha aumentato il grado di controllo relativo che gli stati periferici - sia del Sud che del Nord - possono esercitare sui processi decisionali primari. È sufficiente fare due esempi. Il primo riguarda i motivi per cui i paesi periferici sono favorevoli alla creazione di una banca centrale europea e di una moneta unica. Come hanno affermato le autorità irlandesi in seguito alla svalutazione della lira irlandese nel febbraio del 1993, una banca centrale europea darebbe a tutti i paesi membri, a prescindere dalle loro dimensioni, un ruolo più ampio nei processi decisionali riguardanti i tassi di in- 
teresse e le politiche finanziarie a livello europeo. I paesi periferici si trovano attualmente alla mercé di decisioni prese da uno $o$ più paesi del centro in base a considerazioni prettamente nazionali; e queste decisioni non sono soggette ad alcun veto, dibattito o modifica mediante un processo decisionale collettivo.

In modo analogo, la rotazione della presidenza del Consiglio europeo dà ai paesi più piccoli un ruolo di leadership a livello europeo e persino mondiale che non sarebbe loro concesso se rappresentassero soltanto loro stessi. L'attuazione del Trattato di Maastricht comporterà un maggiore ventaglio di poteri per il Parlamento europeo, e il nuovo Comitato delle regioni permetterà $a$ un insieme di entità politiche e amministrative subnazionali - attualmente inattive a livello comunitario a causa del predominio degli stati nazionali mediante l'importantissimo Consiglio dei ministri - di essere rappresentati e di esprimersi politicamente nei processi decisionali comunitari. In questo modo l'integrazione politica, invece che togliere poteri agli stati periferici, conferisce loro maggiore autorità e prestigio. E sbagliato concepire l'integrazione come una strada a senso unico che avvantaggia i paesi più industrializzati del centro. Piuttosto, essa è un processo che contribuisce alla riduzione delle diseguaglianze economiche e politiche create dal sistema degli stati nazionali.

[traduzione di Giancarlo Gasperoni]

\section{Riferimenti bibliografici}

Arrighi, G. (a cura di) (1985), Semiperipheral Development: The Politics of Southern Europe in the Twentieth Century, London, Sage.

Bei, Banca europea degli investimenti (1991), Annual Report 1990.

Biehl, D., D. Hussman e S. Schnyder (1972), Zur Regionalen Einkommenverteilung in der Europaisch Wirtschaftsgemeinschaft, in «Die Weltwirtschaft», pp. 64-68.

Cec (1990), Second Survey on State Aids in the European Community in the Manufacturing and Certain Other Sectors, Luxembourg, Office of Official Publications of the European Communities.

- (1992), Community Structural Policies: Assessment and Outlook, Com(92) 84 Final.

Cuadrado Roura, J. e L. Suarez-Villa (1992), Regional Economic Integration and the Evolution of Disparities, Fourth World Congress of the Regional Science Association, Palma de Mallorca, 26-30 maggio. 
Haas, E.B. (1958), The Uniting of Europe: Political, Social and Economic Forces, 1950-1957, London, Steven and Sons.

- (1975), The Obsolescence of Integration Theory, Berkeley, Institute of International Studies.

Hudson, R. e J. Lewis (a cura di) (1985), Uneven Development in Southern Europe: Studies of Accumulation, Class, Migration and the State, London, Methuen.

Leonardi, R. (1991), Riflessi della riduzione del sostegno comunitario sull'economia regionale, con particolare riguardo al sistema industriale, Giunta regionale Regione Abruzzo.

- (1993a), The State of Economic and Social Cohesion in the Community Prior to the Creation of the Single Market: The View from the Bottom-Up, Report to the European Commission.

- (1993b), Cohesion in the European Community: Illusion or Reality?, in «Western European Politics», in corso di stampa.

Lindberg, L. (1963), The Political Dynamics of European Economic Integration, London, Oxford University Press.

Madureira-Pires, L. (1992), European Community Development Policies: The Case of Portugal, relazione presentata all'European Research Seminar, London School of Economics, Department of Government, 28 febbraio.

Molle, W., B. van Hoist e H. Smit (1980), Regional Disparity and Economic Development in the European Community, Westmead, Saxon House.

Nanetti, R.Y. (1992), Coordination in Development Planning: An Evaluation of the Initial Implementation of the Community Support Framework, Commission of the European Communities.

Overturf, S.F. (1986), The Economic Principles of European Integration, New York, Praeger.

Putnam, R.D., con la collaborazione di R. Leonardi e R.Y. Nanetti (1993), Making Democracy Work: Civic Traditions in Modern Italy, Princeton, Princeton University Press; trad. it. La tradizione civica nelle regioni italiane, Milano, Mondadori, 1993.

Rokkan, S. e D.W. Urwin (1982) (a cura di), The Politics of Territorial Identity, London, Sage.

- (1983), Economy, Territory and Identity: Politics of Western European Peripheries, London, Sage.

Sartori, G. (1976), Parties and Party Systems: A Framework for Analysis, New York, Cambridge University Press.

Seers, D., B. Schaffer e L. Kiljunen (a cura di) (1979), Underdeveloped Europe: Studies in Core-Periphery Relations, London, Marvoster Press.

Tarrow, S. (1977), Between center and Periphery: Grassroots Politicians in Italy and France, New Haven, Yale University Press.

Urwin, D.W. (1985), The Price of a Kingdom: Territory, Identity and the Centre-Periphery Dimension in Western Europe, in Y. Meny e 
V. Wright (a cura di), Centre-Periphery Relations in Western Europe, London, Allen and Unwin, pp. 151-170.

Williams, A. (a cura di) (1984), Southern Europe Transformed: Political and Economic Change in Greece, Italy, Portugal and Spain, London, Harper and Row. 